\title{
Diversidade de plantas alimentares em quintais agroflorestais de Cuiabá e Várzea Grande, Mato Grosso, Brasil
}

\author{
Diversity of food plants in agroforestry yards of Cuiabá and Várzea Grande, Mato \\ Grosso, Brazil
}

\section{Diversidad de plantas alimentarias en quintos agroforestales de Cuiabá y Várzea Grande, Mato Grosso, Brasil}

\author{
Renan Nunes Bortoluzzi ${ }^{1}$ \\ Lineuza Leite Moreira ${ }^{1}$ \\ Cristiane Ramos Vieira ${ }^{1}$
}

\begin{abstract}
Resumo: A pesquisa foi realizada com os ribeirinhos de Cuiabá e Várzea Grande, MT, com o objetivo de avaliar o perfil florístico com enfoque nas cultivares alimentares em quintais agroflorestais dessas regiões ribeirinhas. Verificou-se a predominância de oito espécies nos quintais agroflorestais: Mangifera indica, Annona squamosa, Carica papaya, Citrus latifolia, Citrus sinensis, Malphighia emarginata, Allium fistulosum e Anacardium occidentale.
\end{abstract}

Palavras-chave: culturas alimentares; população ribeirinha; etnobotânica; conhecimento tradicional.

Abstract: The research was carried out with the riverside people in Cuiabá and Várzea Grande, MT, to evaluate the floristic profile aiming at the food cultivars in agroforestry yards of these riverside regions. There was a predominance of eight species in agroforestry yards: Mangifera indica, Annona squamosa, Carica papaya, Citrus latifolia, Citrus sinensis, Malphighia emarginata, Allium fistulosum, and Anacardium occidentale.

Keywords: food cultures; riverside population; ethnobotany; traditional knowledge.

Resumen: La investigación fue realizada con los ribereños en Cuiabá y Várzea Grande, MT, con el objetivo de evaluar el perfil florístico con enfoque en las cultivares alimenticias en quintos agroforestales de esas regiones ribereñas. Hubo un predominio de ocho especies en los quintos agroforestales: Mangifera indica, Annona squamosa, Carica papaya, Citrus latifolia, Citrus sinensis, Malphighia emarginata, Allium fistulosum y Anacardium occidentale.

Palabras clave: culturas alimentarias; población ribereña; etnobotánica; conocimiento tradicional.

\section{INTRODUÇÃO}

A produção de alimentos sempre esteve relacionada ao meio rural; entretanto, com o advento da modernização, houve a migração do meio rural para o urbano. É válido ressaltar que, mesmo com essa transição, a população não perdeu o hábito de plantar.

Com espaços livres na parte externa da casa, denominados quintais, criam plantas de todos os portes para todos os fins. Chagas et al. (2014) define os quintais como áreas produtivas localizadas próximas às residências; além dessa consideração, os quintais podem ser vistos como espaços nos quais se manifestam relações com o mundo natural, simbólico e social, possibilitando estudos etnobiológicos (POSEY, 1987).

Roças e quintais apresentam diversidade de espécies vegetais, as quais são utilizadas e manejadas pelos agricultores, por populações locais, para garantir a oferta de produtos alimentícios e medicinais para subsistência, assim como para geração de renda das famílias (DUARTE; PASA, 2016).

\footnotetext{
${ }^{1}$ Universidade de Cuiabá (UNIC), Cuiabá, Mato Grosso, Brasil.
} 
Os quintais exercem um papel importante para a segurança alimentar dos agricultores familiares (SOUZA et al., 2017). Partindo dessas constatações, essas áreas ao redor das casas dão alimento, promovem ambiência, geram renda e são mantenedoras de tradições e ritos com os recursos naturais (LOBATO et al., 2017). Autores como Ferreira e Dias (1993) e Amorozo (2002) destacam que os quintais se constituem como espaços nos quais espécies raras podem ser encontradas e como locais remanescentes da composição florística local.

As áreas plantadas representam ainda, segundo Medeiros et al. (2004), a identidade de uma população, ao passo que a vegetação ali plantada reflete o que as pessoas pensam e o que são, além do estabelecimento de um vínculo com o meio do qual fazem parte. A diversidade de espécies encontradas nos quintais e as interações que são estabelecidas pelas pessoas que as plantam transcorrem pelo caráter econômico, social e cultural que esse espaço representa (KUMAR; NAIR, 2006).

Os conhecimentos tradicionais sobre o uso e a conservação da agrobiodiversidade - a agrobiodiversidade pode ser definida como a parcela da biodiversidade utilizada direta ou indiretamente para a alimentação e agricultura, abrangendo a diversidade de plantas, animais e microrganismos, incluindo o aproveitamento dos recursos para fins medicinais, fibra, combustível, entre outros (ALMEIDA; PASA; GUARIM, 2014) - nos quintais são parte das diversas estratégias agroalimentares que as comunidades tradicionais criam para manter e garantir a alimentação (PEREIRA et al., 2017).

Nas comunidades ribeirinhas do Rio Cuiabá, os quintais representaram o alicerce da produção de alimentos que sustentaram as famílias por muitos anos. Diante disso, Duarte e Pasa (2016) enfatizam que é necessário resgatar os sistemas de manejo adotados por essas populações, pois já se sabe que essas técnicas contribuem significativamente para a manutenção da agrobiodiversidade.

Nessa perspectiva, uma das formas de se fazer esse resgate é por meio do conhecimento etnobotânico - a etnobotânica dedica-se a estudar a estrutura de comunidades vegetais e paisagens que são manejadas pelas pessoas no seu cotidiano (DAVID; PASA, 2017).

Pesquisas etnobotânicas têm exposto ao público e à comunidade científica a importância cultural das plantas para diferentes povos e comunidades tradicionais (CARNEIRO; BARBOZA; MENEZES, 2010). Logo, destaca-se a importância de estudos interdisciplinares com enfoque na análise e interpretação do conhecimento, das formas de manejo e de uso e do valor cultural, em pesquisas etnobotânicas. (PASA; NEVES; ALCÂNTARA, 2008). As autoras destacam que se faz necessário o conhecimento das relações que as sociedades estabelecem com seu meio ambiente, assim como a compreensão dos princípios genéricos de funcionamento e da dinâmica dos sistemas ecológicos.

Uma das comunidades ribeirinhas estudadas é a de Bonsucesso, distrito mais antigo de Várzea Grande, MT, criada em 1823, que cresceu aos arredores de um engenho de açúcar. Os ribeirinhos locais, atualmente, vivem economicamente pela venda de peixe, pelos artesanatos como redes bordadas, horticultura, cana-de-açúcar, produção de doce e rapadura pela arte artesanal (SILVA, 2011).

A comunidade São Gonçalo Beira Rio foi estabelecida no século XVIII, à margem esquerda do Rio Cuiabá. Essa comunidade vive do artesanato em cerâmica e manifesta sua cultura por meio de danças como o cururu, o siriri e a roda de São Gonçalo, e de suas comidas típicas (NUNES; ROMANCINI, 2004). 
A comunidade Passagem da Conceição está situada no espaço mais encachoeirado de Cuiabá acima, nos extremos dos limites cuiabanos com Várzea Grande. A comunidade tem como limites, ao Sul e ao Leste, o Rio Pari; e, ao Norte e Oeste, parte das linhas divisórias do município de Nossa Senhora do Livramento e, na íntegra, as de Acorizal (RIBEIRO; GUARIM NETO; PASA, 2011).

As pesquisas mato-grossenses sobre quintais demonstram um consenso quanto à utilização das plantas em três categorias principais: alimentação, medicina popular e ornamentação (AMARAL et al., 2017). No entanto, torna-se relevante destacar que os estudos que abrangem tal assunto são limitados. Ao se ter conhecimento dessas particularidades, estas tornaram-se inquietações, que despertaram o interesse na realização deste trabalho no intuito de avaliar o perfil florístico com enfoque nas cultivares alimentares em quintais das regiões ribeirinhas nos municípios de Cuiabá e Várzea Grande, MT, verificando a distribuição das famílias das cultivares alimentares.

\section{MATERIAL E MÉTODOS}

O estudo foi realizado nas cidades de Cuiabá e Várzea Grande, Mato Grosso, nas comunidades ribeirinhas de Bonsucesso e Passagem da Conceição, em Várzea Grande; e São Gonçalo Beira Rio, em Cuiabá. São Gonçalo localiza-se a 1535'56" latitude S e 56006'01"

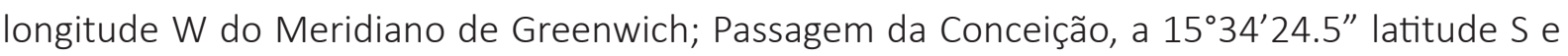
$56^{\circ} 09^{\prime} 00.8^{\prime \prime}$ longitude W; e Bonsucesso, a 1543'05.8" latitude S e 56 06'46.0" longitude W. Ao todo, foram visitadas 62 residências, 19 na comunidade São Gonçalo, 30 em Bonsucesso e 13 em Passagem da Conceição.

O estudo foi dividido em dois momentos: o primeiro se deu a partir da aplicação de questionário aos moradores, e o segundo contemplou o estudo em bibliografias que abordam as temáticas relacionadas ao estilo de vida de moradores antigos que se mantiveram às margens dos rios Cuiabá e Coxipó, bem como para a identificação das espécies encontradas, tendo como auxílio a literatura de Silva et al. (1995), Lorenzi e Matos (2002) e Souza e Lorenzi (2005).

No primeiro momento e para maior aproveitamento e efetividade do estudo, foram estabelecidas perguntas dissertativas e outras com possibilidades de escolhas, contendo tópicos com questões referentes à etnobotânica, dados socioeconômicos (idade, sexo, procedência), dados referentes à diversidade das plantas presentes nos quintais das residências de cada entrevistado; dados botânicos (nome popular da planta) e populares; e de métodos de manejo (adubação) das espécies plantadas.

As questões com múltiplas escolhas foram analisadas com base na frequência com que o item foi assinalado pelo pesquisador, enquanto as questões abertas foram analisadas de acordo com as respostas dos participantes, formando, assim, categorias de respostas para melhor representação delas.

Para responder à pesquisa, os participantes deveriam ser residentes das Comunidades de Bonsucesso, Passagem da Conceição ou São Gonçalo Beira Rio. Participaram aqueles que manifestaram interesse em colaborar com a pesquisa e conforme disponibilidade de tempo em responder às questões, totalizando 62 ribeirinhos. As casas que são utilizadas apenas para fins de semana não foram consideradas.

Para análise dos dados, foram calculados os índices de ocorrência e dominância segundo classificação proposta por Palma (1975). A ocorrência das espécies foi agrupada em três classes: 
acidental $(0,0$ a 25,0\%), acessória $(25,0$ a 50,0\%) e constante $(50,0$ a 100,0\%). 0 índice de dominância também estabeleceu três classes: acidental (0,0 a 2,5\%), acessória (2,5 a 5,0\%) e dominante (5,0 a 10,0\%). A combinação destes dois índices permitiu classificar as espécies em: comum, quando constante e dominante; intermediário, quando constante e acessório, constante e acidental, acessório e acidental, acessório e dominante, acessório e acessório; e raro, quando acidental e acidental.

O índice de ocorrência (IO) foi calculado da seguinte forma:

$$
\mathrm{IO}=\frac{\text { Número de amostras onde foi registrada a espécie }}{\text { Número de amostras de cada local }} \times 100
$$

O índice de dominância foi dado da seguinte forma:

$$
\text { ID }=\frac{\text { Número de indivíduos da espécie }}{\text { Número total de indivíduos }} \times 100
$$

\section{RESULTADOS E DISCUSSÃO}

Em Bonsucesso, Passagem da Conceição e São Gonçalo Beira Rio, foram encontrados um total de 700 indivíduos de plantas alimentares. Em relação ao número de indivíduos e de espécies em cada bairro, foram encontrados, respectivamente, 334 e 71 em Bonsucesso; 139 e 49 em Passagem da Conceição; e 226 e 64 em São Gonçalo Beira Rio.

Em Bonsucesso, as espécies observadas foram distribuídas em 37 famílias (Figura 1); em Passagem da Conceição, em 31 (Figura 2); e em São Gonçalo Beira Rio, em 34 famílias (Figura 3).

No bairro de Bonsucesso, foram encontrados 334 indivíduos pertencentes a 37 famílias: Anacardiaceae (54); Rutaceae (45); Myrtaceae (25); Solanaceae (22); Apiaceae (20); Liliaceae (19); Musaceae (18), Malpighiaceae (14); Lamiaceae (12); Arecaceae (9); Asteraceae (8); Caricaceae e Oleraceae (7); Annonaceae, Curcubitaceae, Euphorbiaceae, Oxalidaceae e Poaceae (6); Lauraceae (5); Fabaceae, Malvaceae, Moraceae e Rubiaceae (4), Lythraceae, Passifloraceae e Sapindaceae (3); Acanthaceae, Brassicaceae e Bromeliceae (2) e as demais famílias (8) com uma espécie cada uma.

As famílias que apresentaram maior riqueza de espécies nos quintais estudados na Comunidade de Cuiabá Mirim, por Almeida, Rodrigues e Norder, (2014), foram: Anacardiaceae (5 espécies), Arecaceae, Fabaceae, Lamiaceae, Malvaceae e Rubiaceae (4 espécies cada uma), Annonaceae, Asteraceae, Myrtaceae, Poaceae e Solanaceae (3 espécies cada uma), sendo que o número de espécies encontrado por quintal variou de 9 a 38 espécies, com uma média de 18 espécies por propriedade. 
Figura 1 - Número de indivíduos de cada família de plantas alimentares encontradas em Bonsucesso

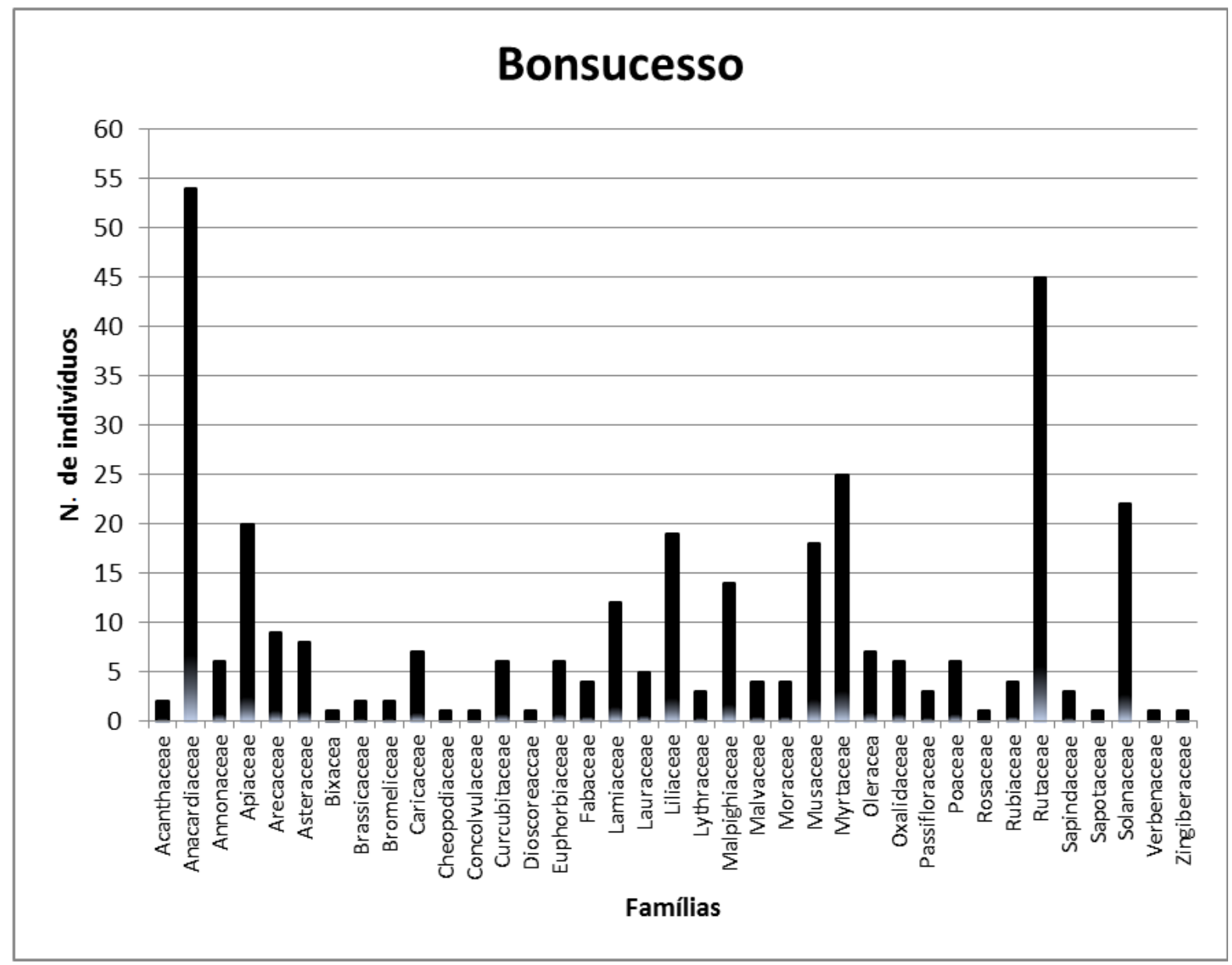

Fonte: Bortoluzzi, Moreira e Vieira (2019).

Em Passagem da Conceição, foram 139 indivíduos distribuídos em 31 famílias: Rutaceae (23); Anacardiaceae (21); Malpighiaceae (11); Myrtaceae (9); Apiaceae (8); Annonaceae, Caricaceae e Poaceae (7); Aracaceae e Liliaceae (6); Malvaceae (5); Fabaceae e Musaceae (3); Bromeliceae, Lamiaceae, Lauraceae, Moraceae e Verbenaceae (2); e as demais famílias (13) com uma espécie cada uma.

No Bairro São Gonçalo Beira Rio, foram observados 226 indivíduos distribuídos em 34 famílias: Anacardiaceae (38); Rutaceae (18); Apiaceae (17), Arecaceae e Malpighiaceae (16); Myrtaceae (12), Liliaceae (11); Lamiaceae, Musaceae e Poaceae (9); Annonaceae e Solanaceae (8); Caricaceae (7), Asteraceae (6); Oxalidaceae (5); Euphorbiaceae e Malvaceae (4); Fabaceae, Oleraceae e Rubiaceae (3); Amaranthaceae, Brassicaceae, Curcubitaceae, Lauraceae, Lythraceae e Sapindaceae (2); e as demais famílias (8) com uma espécie cada uma.

Amaral et al. (2017) identificaram, nos quintais da Baixada Cuiabana, 136 espécies vegetais, cujas famílias botânicas mais representativas foram Fabaceae (13 espécies) e Solanaceae ( 9 espécies), seguidas das famílias Asteraceae, Rutaceae e Poaceae, com seis representantes cada uma. 
Figura 2 - Número de indivíduos de cada família de plantas alimentares encontradas em Passagem da Conceição

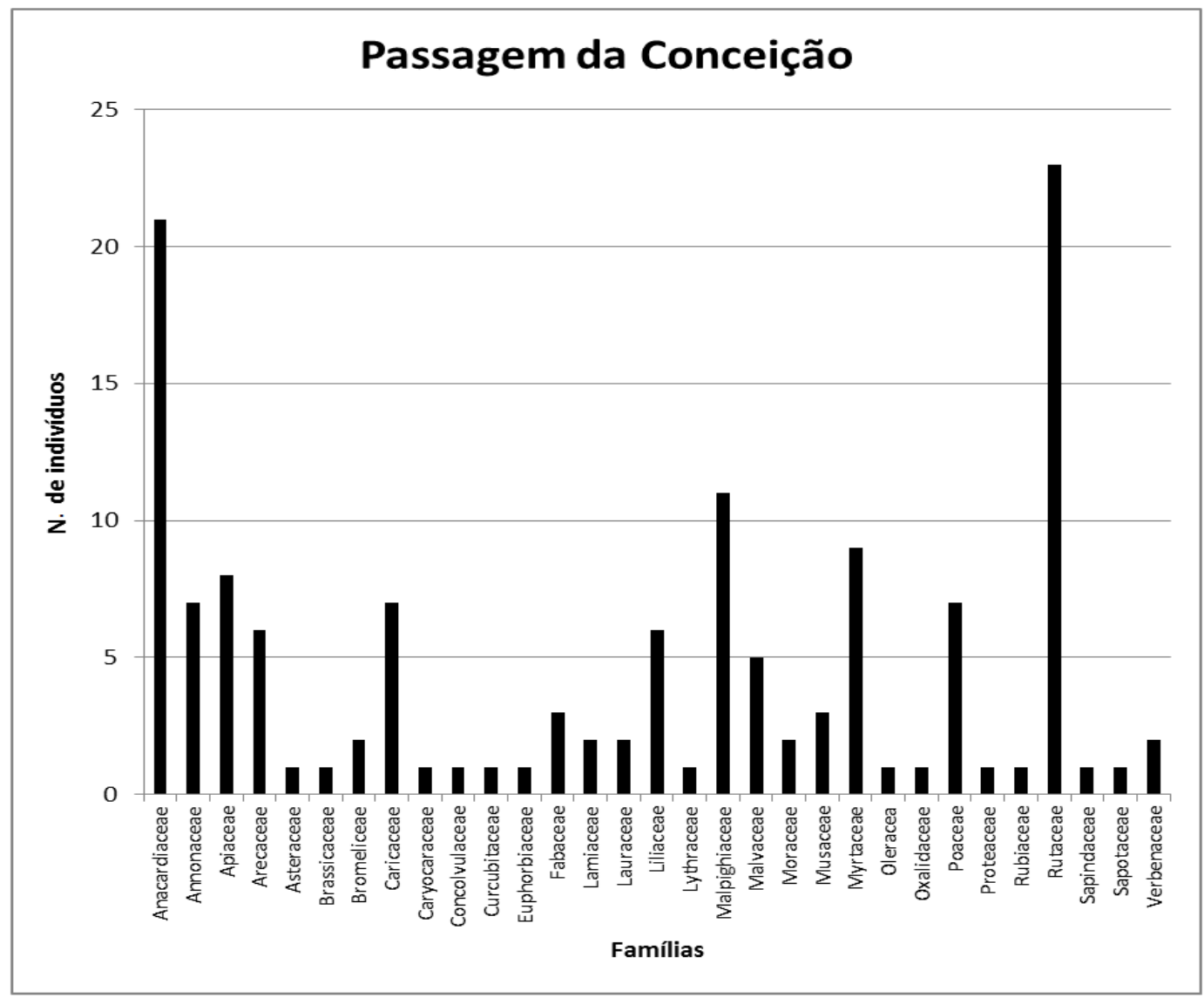

Fonte: Bortoluzzi, Moreira e Vieira (2019).

Figura 3 - Número de indivíduos de cada família de plantas alimentares encontradas em São Gonçalo Beira Rio

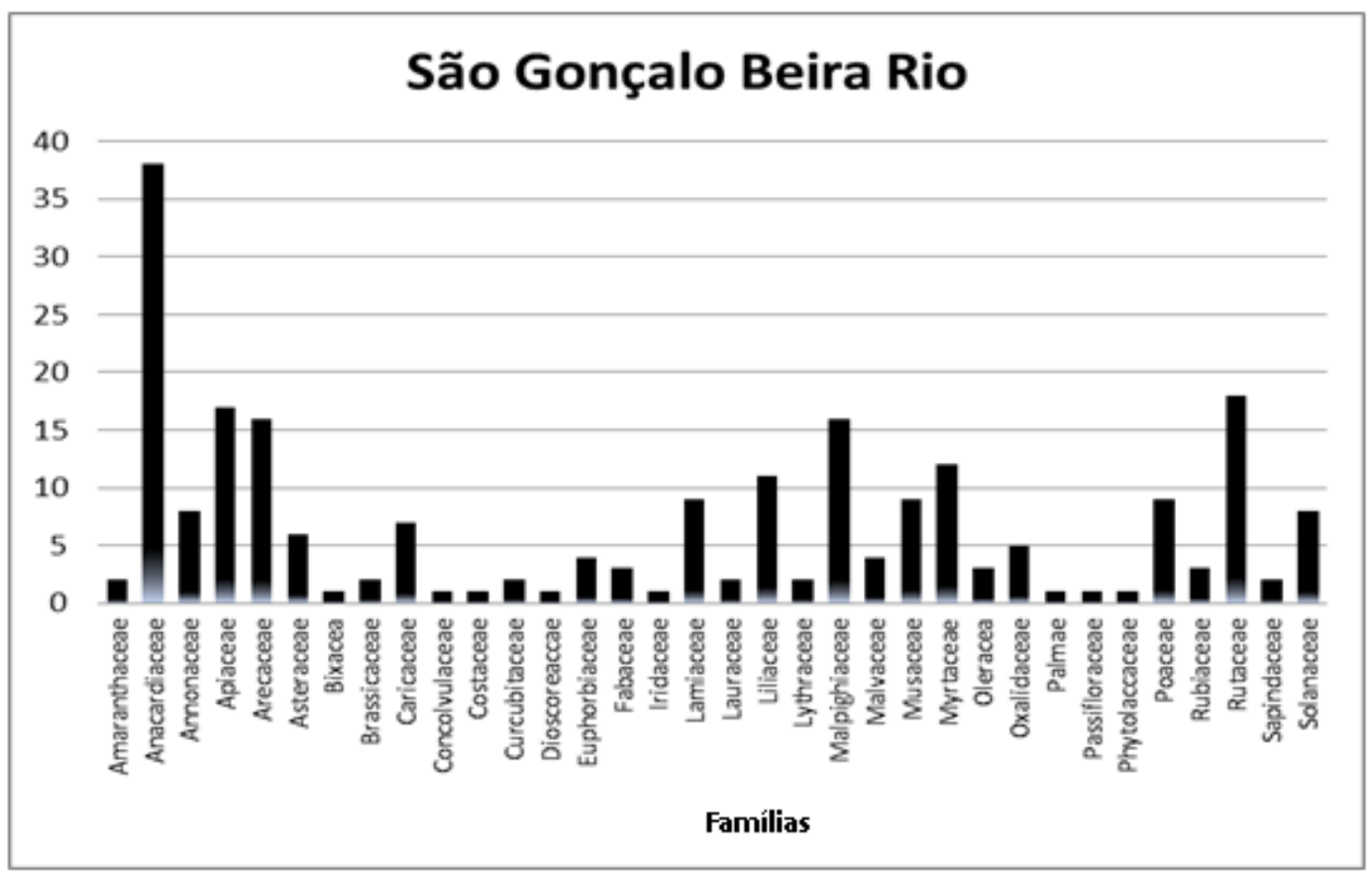

Fonte: Bortoluzzi, Moreira e Vieira (2019). 
Gonçalves e Lucas (2017) identificaram 152 espécies de plantas pertencentes a 121 gêneros e 58 famílias, classificadas nas categorias medicinal, condimentar, alimentar, mística, madeira e outros usos, como para sombra e artesanal, em quintais de Abaetetuba, Pará. O maior número de representantes foi nas famílias Lamiaceae (19\%) e Arecaceae (15,5\%), que se destacaram para fins terapêuticos e alimentares, respectivamente. Já Pereira e Coelho-Ferreira (2017) identificaram espécies de 68 gêneros e 34 famílias botânicas, entre as quais se destacaram Asteraceae (10), Lamiaceae (10), Fabaceae (9), Euphorbiaceae (4), Rutaceae (4), Apocynaceae (3), Arecaceae (3) e Zingiberaceae (3), em Abaetetuba, no Pará.

Analisando as famílias de plantas alimentares encontradas nos bairros Bonsucesso, Passagem da Conceição e São Gonçalo Beira Rio, observou-se a predominância de duas famílias com as maiores riquezas, que foram a Anacardiaceae (113) e a Rutaceae (86).

Dessa forma, verificou-se que os quintais e as espécies alimentícias são, para os ribeirinhos, um fator extremamente importante, com funções de complementação alimentar, fonte de renda (direta e indiretamente), lugar de lazer, de serviços e fonte de qualidade de vida com o melhoramento do microclima do local e da paisagem, proporcionada pelas árvores.

No seu levantamento, Costa et al. (2017) identificaram 42 famílias botânicas, 79 gêneros, com a riqueza de 89 espécies e 908 indivíduos. Quanto ao tipo de uso das espécies, foi dada a seguinte distribuição: alimentar (51), medicinal (13), ornamental (8), madeireira (3), não madeireira (1) sombreamento (9) e condimentos (4). As famílias botânicas com maior abundância identificadas foram a Malvaceae, com 142 indivíduos; a Musaceae e a Lamiaceae, ambas com 80; Arecaceae, com 77; a Euphorbiaceae, 70; Poaceae, 54; e Anacardiaceae, com 53.

De acordo com o Índice de Ocorrência de Palma, no bairro de Bonsucesso, seis indivíduos apresentaram ocorrência constante; seis indivíduos, ocorrência acessória; e 59 indivíduos tiveram ocorrência acidental. Em Passagem da Conceição, seis espécies apresentaram ocorrência constante; seis tiveram ocorrência acessória; e 38 indivíduos, ocorrência acidental. Já em São Gonçalo Beira Rio, seis indivíduos apresentaram ocorrência constante; 11 indivíduos, ocorrência acessória; e 47 indivíduos tiveram ocorrência acidental.

Em relação à dominância das espécies, em Bonsucesso, cinco indivíduos apresentaram ocorrência dominante; quatro indivíduos, ocorrência acessória; e 62 indivíduos apresentaram ocorrência acidental. Em Passagem da Conceição, sete indivíduos apresentaram ocorrência dominante; sete, acessória; e 49 indivíduos apresentaram ocorrência acidental.

A Tabela 1 apresenta a classificação dos indivíduos de acordo com a classificação proposta por Palma (1975).

Tabela 1 - Número e classificação das espécies encontradas nos bairros: nas comunidades ribeirinhas de Bonsucesso e Passagem da Conceição, em Várzea Grande; e São Gonçalo Beira Rio, em Cuiabá

\begin{tabular}{lcccc}
\hline Bairros/Classificação & Comum & Intermediário & Raro & Total \\
\hline Bonsucesso & $7 \%$ & $10 \%$ & $83 \%$ & \\
Passagem da Conceição & $12 \%$ & $14 \%$ & $74 \%$ & \\
São Gonçalo & $3 \%$ & $22 \%$ & $75 \%$ & \\
\hline
\end{tabular}

Fonte: Bortoluzzi, Moreira e Vieira (2019). 
Dentro do bairro de Bonsucesso, $83 \%$ dos indivíduos foram considerados raros; $10 \%$, intermediários; e 7\%, comuns, sendo os indivíduos comuns: Allium fistulosum (cebolinha), Anacardium occidentale (caju), Citrus sinensis (laranja doce), Mangifera indica (manga) e Musa spp (banana). Destacaram-se, portanto, espécies que contribuem para a alimentação familiar, pertencentes às categorias de hortaliças e frutíferas. As verduras, frutas e legumes constituem valiosas fontes de nutrientes para a família e uma alternativa econômica para o consumo de produtos em épocas de crise, a qual, de outra maneira, seria de difícil obtenção (VALADÃO; AMOROZO; MOTTA, 2006).

Em Passagem da Conceição, $74 \%$ dos indivíduos constituíram a classe dos raros; $14 \%$, intermediários; e 12\% foram considerados comuns. Esses indivíduos comuns foram: Annona squamosa (pinha), Carica papaya (mamão), Citrus latifolia (limão-taiti), Citrus sinensis, Malphighia emarginata (acerola) e Mangifera indica. Nesse caso, as espécies frutíferas foram as mais comuns nesses quintais.

Em São Gonçalo Beira Rio, 75\% dos indivíduos constituíram a classe dos raros; $22 \%$, dos intermediários; e 3\% foram considerados comuns. Estes indivíduos comuns foram: Mangifera indica e Malphighia emarginata. Portanto, assim como o que ocorreu em Passagem da Conceição, as frutíferas foram as que se destacaram.

Um estudo de Quaresma et al. (2015) em quintais agroflorestais no nordeste paraense verificou que as plantas frutíferas somaram $30 \%$ do total de espécies. A preferência por essas espécies está relacionada à alimentação das famílias, pois são alimentos frescos e de acesso imediato, diferentemente dos alimentos comprados, de que não se tem conhecimento do tempo, do modo de armazenamento e da circulação, além do próprio processo produtivo, sobre o qual não se sabe dos teores de agrotóxicos (SANTILLI, 2009).

Amaral et al. (2017) destacaram a importância das plantas cultivadas em quintais para a alimentação das famílias, especialmente a acerola (Malpighia glabra), goiabeira (Psidium guajava), laranjeira (Citrus aurantium) e a mangueira (Mangifera indica), frutíferas amplamente citadas, encontradas em mais de $40 \%$ dos quintais pesquisados e com uma concordância quanto ao uso principal acima de $80 \%$.

Lobato et al. (2017), estudando sobre os quintais urbanos de Abaetetuba, observaram que as plantas são cultivadas em $28,4 \%$ dos quintais, sendo que foram registradas 132 espécies vegetais, de 53 famílias botânicas, distribuídas nas categorias frutífera, medicinal, ornamental e condimentar. As frutiferas foram representadas principalmente por Euterpe oleracea $(6,8 \%)$; Mangifera indica (5,2\%); Cocos nucifera (4,5\%); Malpighia punicifolia (3,6\%); Citrus sinensis (2,0\%); e Musa paradisíaca (3,4\%).

Em estudo sobre os quintais do município de Cáceres, Pereira e Figueiredo Neto (2015) constataram que mais de $60 \%$ da população conhece e utiliza plantas como Mangifera indica (manga), Annona squamosa (ata) e Malpighia glabra (acerola) para a alimentação, sendo estas as mais comuns nos quintais analisados - espécies semelhantes às observadas na região de Várzea Grande e de Cuiabá, avaliadas no presente estudo.

O indivíduo mais constante, dominante e comum entre os bairros estudados foi a manga (Mangifera indica), que é um dos frutos mais consumidos no mundo, na forma in natura ou como polpa, suco, néctar, doce e geleia. O alto consumo mundial de manga é atribuído às suas características sensoriais, como o aroma, o qual é um atributo crucial para boa aceitação de qualquer fruta. Também estão como os indivíduos mais comuns: pinha (Annona squamosa), 
mamão (Carica papaya), limão (Citrus latifólia), laranja (Citrus sinensis), acerola (Malphighia emarginata), cebolinha (Allium fistulosum) e caju (Anacardium occidentale).

Coelho et al. (2016) verificaram também a predominância de Carica papaya e Mangifera indica, presentes em 8 e 7 quintais, respectivamente, da região de Mossoró, no Rio Grande do Norte, indicando a importância das espécies frutíferas para as famílias e sua alimentação.

Gonçalves e Lucas (2017) observaram que, do total das espécies registradas nos quintais, 45 (25,3\%) são de usos variados, como o açaí (Euterpe oleracea), a goiaba (Psidium guajava), a goiaba-araçá (Psidium acutangulum), o caju (Anacardium occidentale) e o cupuaçu (Theobrom agrandiflorum). Essas plantas, além de alimentares, sendo consumidas in natura ou em doces e sucos, apresentam uso medicinal em chás e garrafadas, para problemas nos rins (açaí), dores no estômago e diarreia (goiaba e goiaba-araçá), infecções em geral (caju) e gastrite (cupuaçu). As plantas medicinais estão presentes em $80,6 \%$ dos quintais peridomésticos, com 108 espécies, que correspondem a 57,5\% do total. Com elevada citação, destacou-se a hortelã (Mentha sp.) (21) e a babosa (Aloe vera) (17).

A heterogeneidade das plantas dentro dos quintais se dá pela necessidade de alimentos diversificados na alimentação dos ribeirinhos. Conforme Kumar e Nair (2006), o grande número de espécies para fins alimentícios pode justificar a segurança alimentar como o principal objetivo destes agroecossistemas, seja pela quantidade de indivíduos, seja pela qualidade destes alimentos e sua disponibilidade ao longo do ano.

Entre as espécies alimentares, foram encontradas poucas espécies de mata ciliar nos quintais dos ribeirinhos, porque estes não têm o hábito de consumir produtos das plantas de mata ciliar.

Notou-se que o quintal exerce várias funções, não apenas o de fornecer alimento por meio de plantas, mas também como local para lavar roupas, preparar alimento, limpar peixe, local para as crianças brincarem, para fazer reuniões e realizar festas. Os quintais possibilitam encontro entre vizinhos, e muitos utilizam o espaço também para formar um forno, fogão a lenha, abrigo para animais domésticos, depósito de barco e equipamentos de pesca.

Pasa e Ávila (2010) afirmam que o quintal tem uma função econômica favorável na vida dos ribeirinhos. A mão de obra é dominantemente familiar: pai, mãe e filhos. Nos fundos das casas, há o cultivo de pomares que atende à demanda familiar. Afirmam também que esses pomares têm espécies frutíferas de vários tipos e várias regiões, que servem como suplemento alimentar da família.

Apesar da participação de toda a família na implantação e na condução dos quintais, as mulheres têm uma grande relevância no manejo dos quintais, sendo responsáveis pela maior parte das atividades desenvolvidas neles. Portanto, dentro da família, a mulher é a que mais maneja os quintais e as plantas. Gonçalves e Lucas (2017) verificaram também a importância do trabalho das mulheres nos quintais tradicionais. Em pesquisa desses autores, eles observaram que os interlocutores compreenderam a faixa etária entre 19 e 75 anos, sendo a maioria mulheres (47), 70,1\%, e os demais do sexo masculino (20), 29,9\%. De acordo com Novais et al. (2011), em muitos quintais, as espécies medicinais e condimentares ficam aos cuidados das mulheres e, geralmente, estão localizadas próximas à cozinha.

Porém, Pasa e Ávila (2010) afirmam que os demais membros das famílias têm também conhecimentos acerca do cultivo de plantas em quintais; eles dissertam que este conhecimento é transmitido pelos pais e avós ou membros da mesma família. Esse saber tradicional é comum 
dentro dessa cultura e repassado de geração em geração, contraindo o teórico e o prático. No entanto, vários entrevistados da pesquisa realizada por estes autores, bem como no presente caso, demonstraram que a geração mais jovem não se interessa pelo conhecimento e pela experiência dos mais velhos.

O conhecimento popular dos vegetais cultivados nestas áreas é de suma importância para a manutenção das famílias que ali residem, tanto para as atividades mais tradicionais quanto para a comercialização; assim, há uma conservação in situ pelos residentes da comunidade que necessitam destes recursos e os mantêm em suas áreas (GONÇALVES; LUCAS, 2017).

Outra observação que é importante ser ressaltada é que os ribeirinhos têm a cultura de trocar mudas e frutas entre eles, colaborando, assim, para a socialização. Isso também foi constatado por Winklerprins e Oliveira (2010) em estudos com moradores da periferia de Santarém, PA.

A presente pesquisa indicou também que a maioria dos ribeirinhos de Bonsucesso não tem o hábito de adubar as plantas, correspondendo, assim, a 31\% do total. O produto mais utilizado pelos ribeirinhos de Bonsucesso foi o esterco de boi (21\%) e esterco de boi associado com bagaço de cana (18\%).

No bairro da Passagem da Conceição, notou-se que há maior homogeneidade de produtos utilizados pelos ribeirinhos; porém, o esterco de boi com o adubo 10-10-10 apresentou 31\% da preferência e, no bairro de São Gonçalo Beira Rio, o esterco de boi associado com adubo químico 10-10-10 representou 47\% de uso, constituindo-se como o produto mais utilizado pelos ribeirinhos locais. Amaral et al. (2017) observaram, nos quintais da Baixada Cuiabana, que o principal tipo de adubo utilizado é o orgânico, esterco bovino e de galinha (72\%), o adubo químico (calcários e nitrogenados) foi mencionado por apenas $17 \%$ das famílias.

\section{CONSIDERAÇÕES FINAIS}

O manejo de quintais baseia-se em práticas simples e de baixo custo, uma vez que abrange métodos tradicionais de cultivo de plantas, e a adubação se apresenta como um desses métodos de manejo.

Houve a predominância de oito espécies, manga (Mangifera indica), pinha (Annona squamosa), mamão (Carica papaya), limão (Citrus latifólia), laranja (Citrus sinensis), acerola (Malphighia emarginata), cebolinha (Allium fistulosum) e caju (Anacardium occidentale).

A espécie vegetal Mangifera indica foi o indivíduo mais constante, dominante e comum entre os bairros estudados.

\section{REFERÊNCIAS}

ALMEIDA, S. E.; PASA, M. C.; GUARIM, V. L. M. S. Uso da biodiversidade em quintais de comunidades tradicionais da Baía de Chacororé, Barão de Melgaço, Mato Grosso, Brasil. Revista Biodiversidade, Rondonópolis, v. 13, n. 1, p. 141-55, 2014. Disponível em: http://periodicoscientificos.ufmt.br/ojs/index. php/biodiversidade/article/viewFile/1546/1215. Acesso em: 11 nov. 2018.

ALMEIDA, T. V. V.; RODRIGUES, M.; NORDER, L. A. C. Agrobiodiversidade nas comunidades GuaraniNhandewa no norte do Paraná: memória e resgate. Espaço Ameríndio, Porto Alegre, v. 8, n. 1, p. 40-58, 2014. DOI: https://doi.org/10.22456/1982-6524.41066 
AMARAL, C. N.; SOUZA, G. C.; RITTER, M. R.; LOBORUK, N.; MELO, R. S. P. Contribuição dos quintais na conservação do cerrado e da agrobiodiversidade: um estudo dos quintais tradicionais da Baixada Cuiabana. Amazônica, Revista de Antropologia, Belém, v. 9, n. 1, p. 294-314, 2017. DOI: http://dx.doi.org/10.18542/ amazonica.v9i1.5492

AMOROZO, M. C. M. Traditional agriculture, enduring spaces and the joy of planting. In: ALBUQUERQUE, U. P.; ALVES, A. G. C.; SILVA, A. C. B. L.; DA SILVA, V. A. (Org.). Actualities in Ethnobiology and Ethnoecology. Recife: Ed. SBEE, 2002. p. 123-31.

CARNEIRO, D. B.; BARBOZA, M. S. L.; MENEZES, M. P. Plantas nativas úteis na Vila dos Pescadores da Reserva Extrativista Marinha Caeté-Taperaçu, Pará, Brasil. Acta Botânica Brasílica, Belo Horizonte, v. 24, n. 4, p. 1027-33, 2010. DOI: http://dx.doi.org/10.1590/S0102-33062010000400017

CHAGAS, J. C. N.; FRAXE, T. J. P.; ELIAS, M. E. A.; CASTRO, A. P.; VASQUES, M. S. Os sistemas produtivos de plantas medicinais, aromáticas e condimentares nas comunidades São Francisco, Careiro da Várzea e Santa Luzia do Baixio em Iranduba no Amazonas. Revista Brasileira de Agroecologia, Pelotas, v. 9, n. 1, p. $111-$ 21, 2014. Disponível em: http://orgprints.org/26492/1/Chagas_Sistemas.pdf. Acesso em: 11 nov. 2018.

COELHO, M. F. B.; LEAL, C. C. P.; OLIVEIRA, F. N.; NOGUEIRA, N. W.; FREITAS, R. M. O. Levantamento etnobotânico das espécies vegetais em quintais de bairro na cidade de Mossoró, Rio Grande do Norte. Revista Verde de Agroecologia e Desenvolvimento Sustentável, Pombal, v. 11, n. 4, p. 154-62, 2016. DOI: http://dx.doi.org/10.18378/rvads.v11i4.3953

COSTA, G. C.; MOURA, N. D. S.; FARIAS; A. K. D.; ALHO, E. A.; JUCOSKI, G. O. Caracterização socioeconômica e levantamento de espécies vegetais em quintais agroflorestais da zona rural do município de Parauapebas, Pará. Agroecossistemas, Belém, v. 9, n. 1, p. 199-211, 2017. Disponível em: https://periodicos.ufpa.br/ index.php/agroecossistemas/article/viewFile/4653/4399. Acesso em: 11 nov. 2018.

DAVID, M.; PASA, M. C. Ribeirinhos e recursos vegetais: a etnobotânica em Bonsucesso, Várzea Grande, Mato Grosso. FLOVET - Boletim do grupo de pesquisa da flora, vegetação e etnobotânica, Cuiabá, v. 1, n. 8, p. 35-49. 2016. Disponível em: http://periodicoscientificos.ufmt.br/ojs/index.php/flovet/article/ view/4028/2805. Acesso em: 11 nov. 2018.

DUARTE, G. S. D.; PASA, M. C. Agrobiodiversidade e a etnobotânica na comunidade São Benedito, Poconé, Mato Grosso, Brasil. Interações, Campo Grande, v. 17 n. 2, p. 247-56, abr./jun., 2016. DOI: http://dx.doi. org/10.20435/1984042X2016208

FERREIRA, M. S. F. D.; DIAS, F. M. S. Comparison of the usage of space for yards in two neighborhoods at Cuiabá municipality-MT. In: NATIONAL SYMPOSIUM OF ENVIRONMENT STUDIES, 4., 1993, Cuiabá. Anais [...]. Cuiabá, 1993. p. 83-91.

GONÇALVES, J. P.; LUCAS, F. C. A. Agrobiodiversidade e etnoconhecimento em quintais de Abaetetuba, Pará, Brasil. Revista Brasileira de Biociências, Porto Alegre, v. 15, n. 3, p. 119-34, 2017. Disponível em: http://www.ufrgs.br/seerbio/ojs/index.php/rbb/article/view/3756/1362. Acesso em: 11 nov. 2018.

KUMAR, B. M.; NAIR, P. K. R. Tropical homegardens: a time-tested example of sustainable agroforestry. Dordrecht: Springer Science, 2006.

LOBATO, G. J. M.; LUCAS, F. C. A.; TAVARES-MARTINS, A. C. C.; JARDIM, M. A. G.; MARTORANO, L. G. Diversidade de uso e aspectos socioambientais de quintais urbanos em Abaetetuba, Pará, Brasil. Revista Brasileira de Agroecologia, Pelotas, v. 12, n. 2, p. 95-105, 2017. Disponível em: https://ainfo.cnptia. embrapa.br/digital/bitstream/item/162473/1/RBA-Lucieta.pdf. Acesso em: 11 nov. 2018. 
LORENZI, H.; MATOS, F. J. A. Plantas medicinais no Brasil. Nova Odessa: Instituto Plantarum, 2002.

MEDEIROS, M. F. T.; FONSECA, V. S.; ANDREATA, R. H. P. Plantas medicinais e seus usos pelos sitiantes da Reserva Rio das Pedras, Mangaratiba, RJ, Brasil. Acta Botanica Brasilica, Brasília, v. 18, n. 2, p. 391-99, 2004.

NOVAIS, A. M.; GUARIM NETO, G.; GUARIM, V. L. M. S.; PASA, M. C. Os quintais e a flora local: um estudo na comunidade Jardim Paraíso, Cáceres-MT, Brasil. Revista Biodiversidade, Rondonópolis, v. 1, n. 1, p. 1-10, 2011. Disponível em: http://periodicoscientificos.ufmt.br/ojs/index.php/biodiversidade/article/ view/523/445. Acesso em: 11 nov. 2018.

NUNES, P. A. S. S.; ROMANCINI, S. R. Um estudo sobre a produção artesanal na comunidade São Gonçalo Beira Rio, Cuiabá-MT. In: REUNIÃO ANUAL DA SBPC, 56., 2004, Cuiabá. Anais [...]. Cuiabá: SBPC, 2004.

PALMA, S. Contribución al estudio de los sifonoforos encontrados frente a la costa de Valparaiso. Aspectos ecológicos. In: SIMPÓSIO LATINOAMERICANO SOBRE OCEANOGRAFIA BIOLÓGICA, 2., 1975, Venezuela. Anais [...]. Venezuela: Universidad D’Oriente, p.119-133, 1975.

PASA, M. C.; NEVES, W. M. S.; ALCÂNTARA, K. C. Enfoque etnobotânico das categorias de uso das plantas na unidade de paisagem quintal, Comunidade Fazenda Verde em Rondonópolis, MT. Biodiversidade, Cuiabá, MT, v. 7, n. 1, p. 1-11, 2008.

PASA, M. C.; ÁVILA, G. Ribeirinhos e recursos vegetais: a etnobotânica em Rondonópolis, Mato Grosso, Brasil. Interações, Campo Grande, MS, v. 11, n. 2, p. 195-204, 2010. DOI: http://dx.doi.org/10.1590/ S1518-70122010000200008

PEREIRA, L. S.; SOLDATI, G. T.; DUQUE-BRASIL, R.; COELHO, F. M. G.; SCHAEFER, C. E. G. R. Agrobiodiversidade em quintais como estratégia para soberania alimentar no semiárido norte mineiro. Ethnosciencia, Botucatu, v. 2, n. 1, p. 1-25, 2017. DOI: http://dx.doi.org/10.22276/ethnoscientia.v2i1.40

PEREIRA, M. G. S.; COELHO-FERREIRA, M. Uso e diversidade de plantas medicinais em uma comunidade quilombola na Amazônia Oriental, Abaetetuba, Pará. Biota Amazônia, Macapá, v. 7, n. 3, p. 57-68, 2017. DOI: http://dx.doi.org/10.18561/2179-5746/biotaamazonia.v7n3p57-68

PEREIRA, P. V. M.; FIGUEIREDO NETO, L. F. Conservação de espécies florestais: um estudo em quintais agroflorestais no município de Cáceres-MT. Revista Eletrônica em Gestão, Educação e Tecnologia Ambiental, Santa Maria, v. 19, n. 3, p. 783-93, 2015. Disponível em: https://periodicos.ufsm.br/reget/ article/view/18364/pdf. Acesso em: 11 nov. 2018.

POSEY, D. A. Etnobiologia: teoria e prática. In: RIBEIRO, D. (Ed.). Suma Etnológica Brasileira. Petrópolis, RJ: Vozes/FINEP, 1987. p. 15-28.

QUARESMA, A. P.; ALMEIDA, R. H. C.; OLIVEIRA, C. M.; KATO, O. R. Composição florística e faunística de quintais agroflorestais da agricultura familiar no nordeste paraense. Revista Verde de Agroecologia e Desenvolvimento Sustentável, Pombal, v. 10, n. 5, p. 76-84, 2015. DOI: http://dx.doi.org/10.18378/rvads. v10i5.3706

RIBEIRO, R. S.; GUARIM NETO, G.; PASA, M. C. Passagem da conceição: uma comunidade ribeirinha em Movimento em espaço mato-grossense tradicional. Revista Biodiversidade, Rondonópolis, v. 10, n. 1, p. 110-25, 2011. Disponível em: http://periodicoscientificos.ufmt.br/ojs/index.php/biodiversidade/article/ view/430. Acesso em: 11 nov. 2018.

SANTILLI, J. F. R. Agrobiodiversidade e direitos dos agricultores. PUCviva Revista, Perdizes, v. 36, p. 46-55, 2009. Disponível em: https://pib.socioambiental.org/files/file/PIB_institucional/agrobiodiversidade.pdf. Acesso em: 11 nov. 2018. 
SILVA, M. R. A toponímia em Bonsucesso e Pai André no rio Cuiabá-Mato Grosso. 2011. 115p. Dissertação (Mestrado em Estudos Linguísticos) - Universidade Federal de Mato Grosso, Cuiabá, MT, 2011.

SILVA, F. C.; FONSECA, E. P.; SOARES-SILVA, L. H.; MULLER, C.; BIANCHINI, E. Composição florística e fitossociológica do componente arbóreo das florestas ciliares da bacia do rio Tibagi. 3. Fazenda Bom Sucesso, município de Saponema, PR. Acta Botanica Brasilica, Belo Horizonte, v. 9, n. 2, p. 289-302, 1995. DOI: http://dx.doi.org/10.1590/S0102-33061995000200009

SOUZA, A. M. B.; ALVES, K. N. A.; LOBATO, W. T. S.; LEAL, A. J. S.; ALMEIDA, G. M.; SOUZA, A. A. S.; MOTA, A. V. Aspectos da segurança alimentar com base em quintais agroflorestais na comunidade rural de Santa Luzia do Induá no município de Capitão Poço, PA. Agroecossistemas, Belém, v. 9, n. 2, p. 275-87, 2017. DOI: http://dx.doi.org/10.18542/ragros.v9i2.5028

SOUZA, V. C.; LORENZI, H. Botânica Sistemática: guia ilustrado para identificação das famílias de Angiospermas da flora brasileira, baseado em APG II. Nova Odessa: Instituto Plantarum, 2005.

VALADÃO, L. M.; AMOROZO, M. C. M.; MOTTA, D. G. Produção de Alimentos na unidade domiciliar, dieta e estado nutricional: a contribuição dos quintais em um assentamento rural no estado de São Paulo. In: ALBUQUeRQUE, U. P.; ALMEIDA, C.F.B. (Org.). Tópicos em conservação e etnobotânica de plantas alimentícias. Recife: NUPPEA, 2006.

WINKLERPRINS, A.; OLIVEIRA, P. S. S. Urban agriculture in Santarem, Pará, Brazil: diversity and circulation of cultivated plants in urban homegardens. Boletim Museu Paranaense Emílio Goeldi. Ciências Humanas, Belém, v. 5, n. 3, p. 571-85, nov./dez., 2010. DOI: http://dx.doi.org/10.1590/\$1981-81222010000300002

\section{Sobre os autores:}

Renan Nunes Bortoluzzi: Graduado em Agronomia pela Universidade de Cuiabá (UNIC). Engenheiro Agrônomo. E-mail: renan_nnb@hotmail.com. Orcid: http://orcid.org/0000-0003-3517-1363

Lineuza Leite Moreira: Doutora em Educação pela Universidade Federal de Mato Grosso (UFMT), na linha Formação de Professores. Mestre em Agricultura Tropical e graduada em Ciências Biológicas pela UFMT. Docente na Universidade de Cuiabá. E-mail: lineuza@gmail.com, Orcid: http://orcid.org/0000-0002-1432-8854

Cristiane Ramos Vieira: Doutora em Agricultura Tropical e mestre em Ciências Florestais e Ambientais pela Universidade Federal de Mato Grosso (UFMT). Engenheira Florestal. Docente na Universidade de Cuiabá, Cuiabá, MT. E-mail: cris00986@hotmail.com, Orcid: http://orcid.org/0000-0003-1936-1343 
